\title{
Essential steps in managing complicated urologic chronic pain patients: A nursing perspective
}

\author{
Kerri-Lynn Kelly, RN \\ Department of Urology, Kingston Health Sciences Centre, Queen's University, Kingston, ON, Canada
}

Cite as: Can Urol Assoc J 2018;12(6Supp|3):S178-80. htrp://dx.doi.org/10.5489/cuai.5330

\section{Introduction}

The urologic pain population has a poor reputation among healthcare professionals. These patients are often considered needy, time-consuming, and because these conditions are not usually surgically managed, difficult to treat. This is a misconception. With our clinical approach, we have proven that with active listening, education, and individualizing care plans, this patient population can be successfully managed.

\section{What does our urologic chronic pain clinic look like?}

Our nurse-led urologic pain clinic runs alongside the regular clinic. It operates 1.5 days per week and accommodates 12-15 patients. Time allotted per patient is approximately 30-45 minutes for new referrals and 30 minutes for repeat patients. Referrals are made from family practitioners and emergency departments, as well as secondary referrals from urologists and gynecologists. Our recently published study in the Urology Practice Journal revealed that our symptom improvement score was 50-60\%. But overall improvement in quality of life, coping, increased activity, and general happiness was $90 \%$. This article will outline this clinical framework from the nursing perspective. I believe it is our approach to care that dictates our success.

\section{A comprehensive, nurse-led evaluation}

The initial visit begins with a detailed medical/surgical history. We need to look beyond the bladder to identify associated diagnoses that can contribute to pain/discomfort, e.g., irritable bowel syndrome, fibromyalgia, depression, vasectomy, trauma, sexually transmitted infections, vulvodynia etc. Next, we collect a detailed description of the onset of symptoms, location, severity, frequency and nature of the pain/discomfort, and urinary symptoms. A diagram of the female/male anatomy is helpful for the patient to point out

location of the pain. We find that simple questionnaires, such as the Chronic Prostatitis Symptom Index and O'LearySant and Pain Urgency Frequency questionnaire, allow the patient to describe their symptoms and impact. Quality of life questionnaires like the International Consultation of Incontinence Questionnaire (ICIQ), Pelvic Pain Scale, and Symptom Assessment Scale are really not necessary in regular practice, but we use them to help recognize maladaptive coping strategies quickly. They also generate data that we can use in our ongoing research.

\section{Initial care plan carried out by the urology nurse}

From this comprehensive assessment, we begin formulating a care plan with the patient. Remember that most of these patients have being waiting 5-7 years for an appropriate diagnosis. Many have encountered negative experiences with healthcare professionals either by misdiagnosis, failed treatment, or the belief that it's all in their head. Active listening is crucial to the patient's healing process. We encourage them to talk about their experiences and fears. We reiterate that this condition is chronic, perhaps not curable, but can be successfully managed by setting realistic goals.

Our treatment starts with educating the patient about the condition.

There are several great online references for patients and practitioners (Table 1).

Our next step for our bladder hypertensive patients is to introduce the interstitial cystitis (IC) diet. We ask about food triggers. Some patients have already noticed that certain items cause more bladder discomfort. We encourage them to follow the diet for at least three weeks. With our IC/bladder pain syndrome (BPS) patients, we discuss lifestyle changes like avoiding harsh detergents, soaps, and bubble bath, and wearing cotton underwear. We look for other triggers, such as car vibrations, hormones, and high-impact exercise. For our chronic prostatitis/chronic pelvic pain syndrome (CP/CPPS) patients, we review lifestyle changes like correct bike seats and testicular support. We discuss conservative management: heat therapy, donut cushions, herbal supplementation. We 


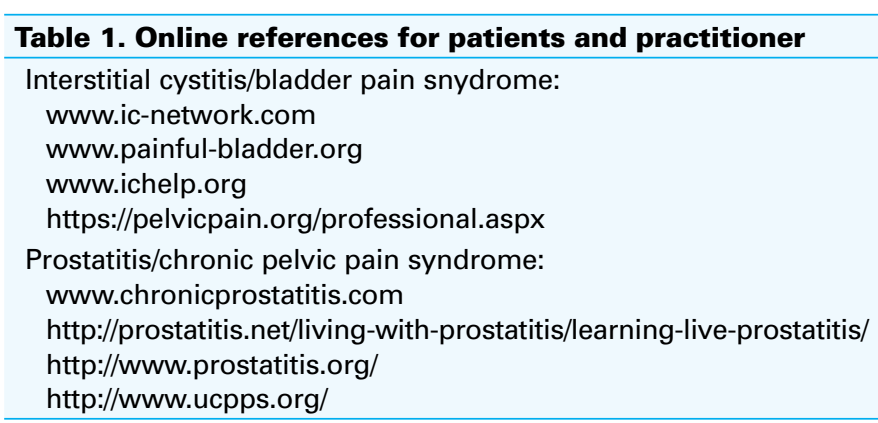

talk about sex with all our patients. We ask if it's uncomfortable and where it hurts: pain with touch and/or penetration. Patients are relieved and appreciative for having us address this subject. This provides us with clues on how to help.

We progress into behaviour management strategies. We encourage low-impact exercise like yoga and tai-chi, and for those who want more vigorous exercise, swimming, walking, and elliptical-low impact exercises. We ask how they cope (or not) with a flare. We have learned in our research that it is very important to identify maladaptive coping strategies like catastrophization (rumination, magnification of symptoms, and helplessness). We know that the presence of these negative ways of thinking directly correlates to decreased response to treatment. We discuss constructive ways to deal with pain, such as mindfulness mediation, journaling, and cognitive behavioural therapy. These conservative management strategies have proven effective in the treatment of IC/BPS and CP/CPPS patients. They are concrete, easy to implement, and give patients control over their condition.

\section{When the does the urologist enter the picture?}

In our clinic process, the urologist now meets the patient. Up to this point, the entire process has been accomplished between the urology nurse and the patient. Sometimes, the urologist is only necessary for confirmation of this type of conserative therapuetic strategy.

The urologist examines the patient with cystoscopy and pelvic exam to accurately diagnose the condition. Cystoscopy will reveal a normal bladder mucosa or abnormalities: Hunner's lesions, inflammation, or glomerulations, etc. It also allows for an appreciation of the functional bladder capacity (usually limited by pain). Sometimes, using an instilled local anesthetic to temporarily ameliorate the bladder pain improves the diagnostic accuracy in determining the involvement of the various potential pain generators. The pelvic exam will identify pain contributors like pelvic floor dysfunction, trigger points, vulvodynia, vaginal atrophy, or prolapse. These findings will determine relevant treatment. For men, the cystoscopy helps rule out lower urinary tract pathology such as strictures, bladder neck hypertrophy, etc. For men, the digital rectal examination (DRE) is performed in clinic, after a midstream urine is collected. The DRE is completed after a prostate massage. We do a post-prostatic massage urine collection following the DRE to help rule out prostate infection.

\section{The management plan comes together}

Further modifications to our symptom management plan are based on the information gathered from the initial visit, cystoscopy, and pelvic exam. We tailor an individual therapy for each unique patient and it almost always involves a multimodal treatment plan. The various medical and surgical therapies employed in our clinic (and those of other specialized urology clinics) are very well-described in previous sections of this supplement.

In our clinic model, pain management is multifaceted. We do not prescribe opioids because we know they do not work for this patient population and actually make it more difficult for the other treatments to work. Instead, we educate the patient on understanding pain. We know that increased stress and anxiety increases the inflammatory response, which activates the immune system, and therefore, increases the pain response. We troubleshoot ways to cope with the pain (conservative management, mindfulness, cognitive behavioural therapy), as well as maintaining a healthy lifestyle during the good times (exercising, diet, laughter, and sleep hygiene). As a third-line of treatment, cannabis has been effective for some patients. It does not help with pain, but does help with coping.

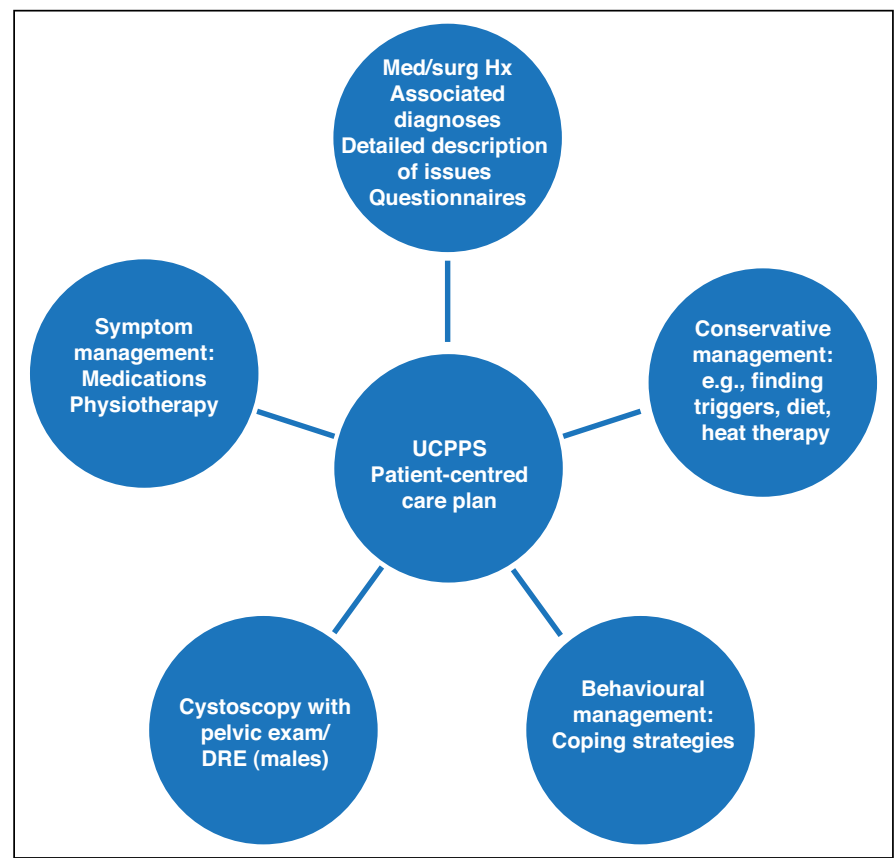

Fig. 1. Urologic chronic pelvic pain syndrome (UCPPS) patient-centred care plan. DRE: digital rectal exam; Hx: history. 


\section{Successful management of urologic chronic pelvic pain syndrome patients involves the urologist and the nurse} (Fig. 1)

Successful management of IC/BPS and CP/CPPS patients must use a biopsychosocial approach. In other words, while medications and surgical approaches advocated by the urologist can be a very important part of the management plan, we have learned that the psychological parameters associated with urologic chronic pain cannot be excluded if we desire a positive outcome. Therapy needs to be multimodal, individualized, and patients must take an active role in their recovery. We need to empower the patient by setting realistic goals (which can incorporate pain, urinary, quality of life, and/or desired activity goals) through diet, lifestyle changes, and behaviour management. We need to look for treatable origins of pain in the bladder and other contributing factors. Lastly, we need to recognize the brain/pain connection and educate our patients on understanding how pain works and appropriate coping strategies. These patients are resilient, strong, and motivated. With the right tools and our guidance, they can be successful in managing their condition.

Competing interests: The author reports no competing personal or financial conflicts related to this work.

This paper has been peer reviewed.

Correspondence: Ms. Kerri-Lynn Kelly, Department of Urology, Kingston Health Sciences Centre, Queen's University, Kingston, 0N, Canada; kellyk1@kgh.kari.net 